\title{
Clinical effects of Lingguizhugan decoction in the treatment of hypertension: a systematic review and meta-analysis
}

\author{
Pingchang Xie ${ }^{1}$, Xinmei $\mathrm{Li}^{2}$, Hua Jiang ${ }^{2}$, Sijie Qiu ${ }^{2}$, Yunyu Liang ${ }^{2}$ \\ ${ }^{1}$ Guangdong Provincial Key Laboratory of Research on Emergency in TCM, Emergency Department, Guangdong Provincial Hospital of Traditional \\ Chinese Medicine, Guangzhou, China; ${ }^{2}$ Geriatrics Department, Guangdong Provincial Hospital of Traditional Chinese Medicine, Guangzhou, \\ China \\ Contributions: (I) Conception and design: Y Liang; (II) Administrative support: P Xie; (III) Provision of study materials or patients: H Jiang; (IV) \\ Collection and assembly of data: X Li; (V) Data analysis and interpretation: S Qiu; (VI) Manuscript writing: All authors; (VII) Final approval of \\ manuscript: All authors. \\ Correspondence to: Yunyu Liang. Department of Geriatrics, Guangdong Provincial Hospital of Traditional Chinese Medicine, Guangzhou 510000, \\ China. Email: wenny2000@163.com.
}

\begin{abstract}
Background Lingguizhugan decoction is commonly used to treat metabolic syndrome; however, its curative effect on hypertension is still unclear. Our study aimed to evaluate the clinical efficacy and safety of Lingguizhugan decoction combined with western medicine in the treatment of hypertension.

Methods: We searched 7 electronic databases for relevant studies and full-text articles involved in the evaluation of clinical effects difference between Lingguizhugan decoction combined with western medicine and western medicine alone. All included articles were quality assessed and data analysis was conducted with Review Manager (5.4). Sensitivity analysis was performed, and the results were visualized by means of forest and funnel plots. Results were expressed as risk ratio (RR) or mean difference (MD), together with their $95 \%$ confidence intervals (CIs).
\end{abstract}

Results: In total, 7 studies eventually met our inclusion criteria. The results showed that Lingguizhugan decoction combined with western medicine had a better improvement in decrease systolic blood pressure (SBP) $(\mathrm{MD}=12.33 \mathrm{mmHg}$; 95\% CI: 3.37 to 21.28; $\mathrm{P}=0.007)$ and diastolic blood pressure (DBP) (MD $=7.42 \mathrm{mmHg}$; 95\% CI: 1.89 to $12.95 ; \mathrm{P}=0.009)$ than western medicine alone, it also had a higher effective ratio $(\mathrm{RR}=1.20 ; 95 \% \mathrm{CI}: 1.11$ to $1.31 ; \mathrm{P}<0.0001)$ and lower adverse reactions $(\mathrm{RR}=0.51 ; 95 \% \mathrm{CI}$ : 0.30 to $0.86 ; \mathrm{P}=0.01)$. The results were robust and no obvious publication bias was observed in this study.

Discussion: Our research supported that Lingguizhugan decoction combined with western medicine can effectively reduce patients' blood pressure and improve their clinical symptoms. Because of the limitation in the quantity and quality of the included studies, further large sample and multi center follow-up controlled trials should be carried out to validate our conclusions.

Keywords: Lingguizhugan decoction; hypertension; western medicine; meta-analysis

Submitted Aug 23, 2021. Accepted for publication Oct 16, 2021.

doi: 10.21037/apm-21-2639

View this article at: https://dx.doi.org/10.21037/apm-21-2639

\section{Introduction}

Hypertension is a progressive cardiovascular syndrome of multiple causes, and the number of people with hypertension globally has recently exceeded 1 billion (1). Hypertension can be divided into primary and secondary according to the exact etiology, of which primary hypertension accounts for about $90 \%(2,3)$. The continuous increase of blood pressure (BP) increases the left ventricular load, which leads to the changes of heart and vascular function and structure over time, and finally leads to left ventricular hypertrophy, ventricular enlargement, heart 
failure, and so on (4-6).

At present, hypertension is predominantly treated with western medicine. Western medicine usually only adopts conventional antihypertensive treatment, from which it is easy to have adverse reactions after long-term use, and the BP can easily rebound after withdrawal. The clinical effect of simple western medicine treatment sometimes cannot meet the needs of patients $(7,8)$. Therefore, actively seeking effective treatment methods is of great significance to improve the curative effect, BP, and blood lipid (9). Through the application of traditional Chinese medicine (TCM) to the treatment of hypertension, the advantages of TCM in reducing BP have been gradually recognized by the majority of doctors and patients $(10,11)$.

Zhongjing Zhang, a famous doctor of the Han Dynasty, mentioned in his book "The typhoid miscellaneous diseases theory" that Lingguizhugan decoction can be used for the treatment of hypertension (12). Lingguizhugan decoction is composed of Poria cocos (Fu Ling), Cinnamon Twig (Gui Zhi), Atractylodes macrocephala (Bai Zhu), and licorice (Gan Cao) and it has the functions of strengthening spleen and promoting drainage $(13,14)$. At present, Lingguizhugan decoction is commonly used to treat chronic bronchitis, bronchial asthma, cardiogenic edema, chronic glomerulonephritis edema, and other diseases (15-17).

There are many meta-analysis on the treatment of hypertension with Chinese and Western medicine, but as the few studies on Lingguizhugan decoction combined with western medicine in the treatment of hypertension, and no relevant meta-analysis has been carried out so far, their overall quality has been uneven due to small sample sizes, and the test quality has not been systematically evaluated. We conducted a meta-analysis on the relevant randomized controlled trials (RCTs) to evaluate the clinical efficacy of Lingguizhugan decoction in the treatment of hypertension, so as to provide reference basis for clinical decision-making. We present the following article in accordance with the PRISMA reporting checklist (available at https://dx.doi. org/10.21037/apm-21-2639).

\section{Methods}

\section{Literature search strategy}

A systematic search of relevant studies was conducted in 7 electronic databases including PubMed, Cochrane Central Register of Controlled Trials (CENTRAL), Embase, China Knowledge Resource Integrated Database (CNKI), Chinese
Biomedical Literature Database (CBM), China Science and Technology Journal Database (VIP) and Wanfang Data. These databases were searched from inception to July 2021. The search was performed by combination of the following search terms using the Boolean operators "OR" and/or "AND": "Lingguizhugan decoction", "hypertension" and "western medicine". There were no restrictions on the publication language in the literature search. The eligibility of titles, abstracts, and studies that were deemed relevant for review of their full texts were independently reviewed and assessed by two authors. Any discrepancies were resolved through discussion with a third author.

\section{Study selection}

The studies had to fulfill the following criteria to be included in the analysis:

(I) Studies comparing patients receiving combination therapy of Lingguizhugan decoction and western medicine (test group) and conventional western medicine alone (control group);

(II) Patients with hypertension [diastolic BP (DBP) $\geq 90 \mathrm{mmHg}$ or systolic BP (SBP) $\geq 140 \mathrm{mmHg}$;

(III) Containing indicators evaluating effectiveness between test group and control group;

(IV) Available in full text.

Studies were excluded for following reasons:

(I) Not meeting the inclusion criteria;

(II) No outcomes of interest were impossible to calculate or extrapolate or were reported;

(III) Articles were reviews, abstracts, letters, case reports, or duplicate publications.

\section{Data extraction and quality assessment}

Study selection, data extraction, and risk of bias assessment were undertaken by two reviewers independently, and any disagreements were resolved by discussion with referral to a third reviewer if required. The following information was extracted from enrolled articles: (I) first author's name; (II) year of publication; (III) study design; (IV) treatment; (V) number of patients (along with gender); (VI) intervention time; (VII) primary outcome. Cochrane bias risk assessment tools were used to evaluate the methodological quality assessment of the included literatures (https://methods. cochrane.org/bias/resources/rob-2-revised-cochrane-riskbias-tool-randomized-trials). 


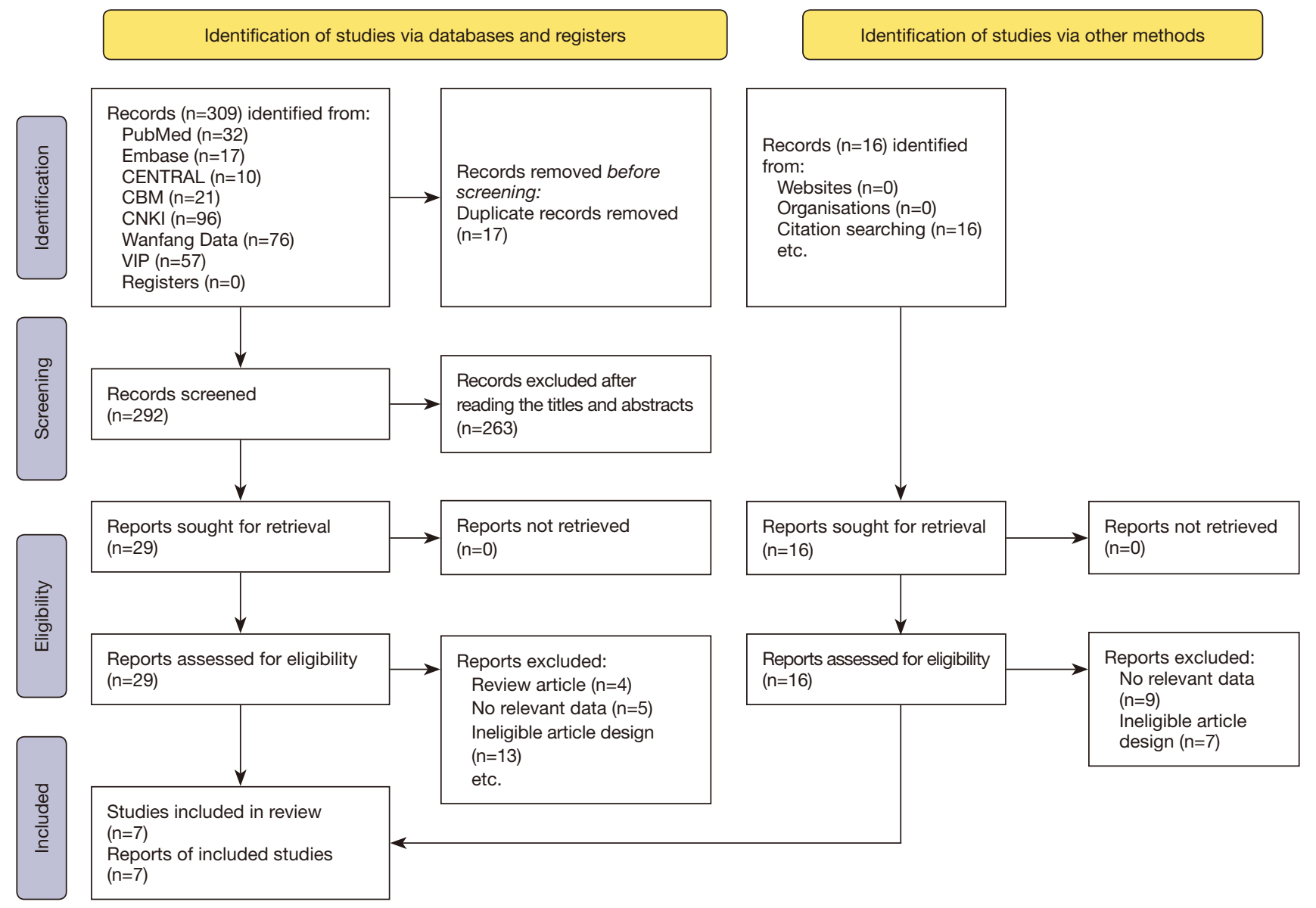

Figure 1 Schematic of the trial selection process.

\section{Statistical analysis}

The software Review Manager (RevMan version 5.4; Cochrane Collaboration, 2020) was used to estimate the impact of the results in the selected studies. All identified articles were included in the systematic review and examined using narrative synthesis. The mean difference (MD) and risk ratio (RR) was used for the measurement data and the count data, and $95 \%$ confidence intervals (CI) were calculated. To assess heterogeneity, we calculated the Cochrane Q statistics and $\mathrm{I}^{2}$ statistics. If $\mathrm{P}<0.05$ or $\mathrm{I}^{2}>50 \%$, indicating a certain degree of heterogeneity among the studies, and the random effects model would be used for analysis; if $\mathrm{P} \geq 0.05$ and $\mathrm{I}^{2} \leq 50 \%$, indicating no heterogeneity or less heterogeneity among the studies, and the fixed effects model was used. We conducted a sensitivity analysis by excluding merged studies one by one and observing whether the synthesis result changed significantly. Funnel plots and Egger's linear regression test were used to discover the publication bias.

\section{Results}

\section{Search process}

In the initial search, we identified a total of 325 studies. After the removal of duplicate files, 308 articles were screened to determine whether they were eligible. After the screening based on the titles and abstracts, 263 articles were excluded. After reading the full text of the remaining 45 articles, 38 studies were further excluded for the following reasons: review article; no relevant data; or ineligible article design. Finally, 7 papers were included in our meta-analysis (18-24). The detailed search process is presented in Figure 1.

\section{Characteristics of included studies}

The detailed characteristics of the 7 eligible studies are summarized in Table 1. The total study sample size was 546 participants (range 60 to 98; Table 1). The duration of 
Table 1 Clinical baseline information of all the included patients

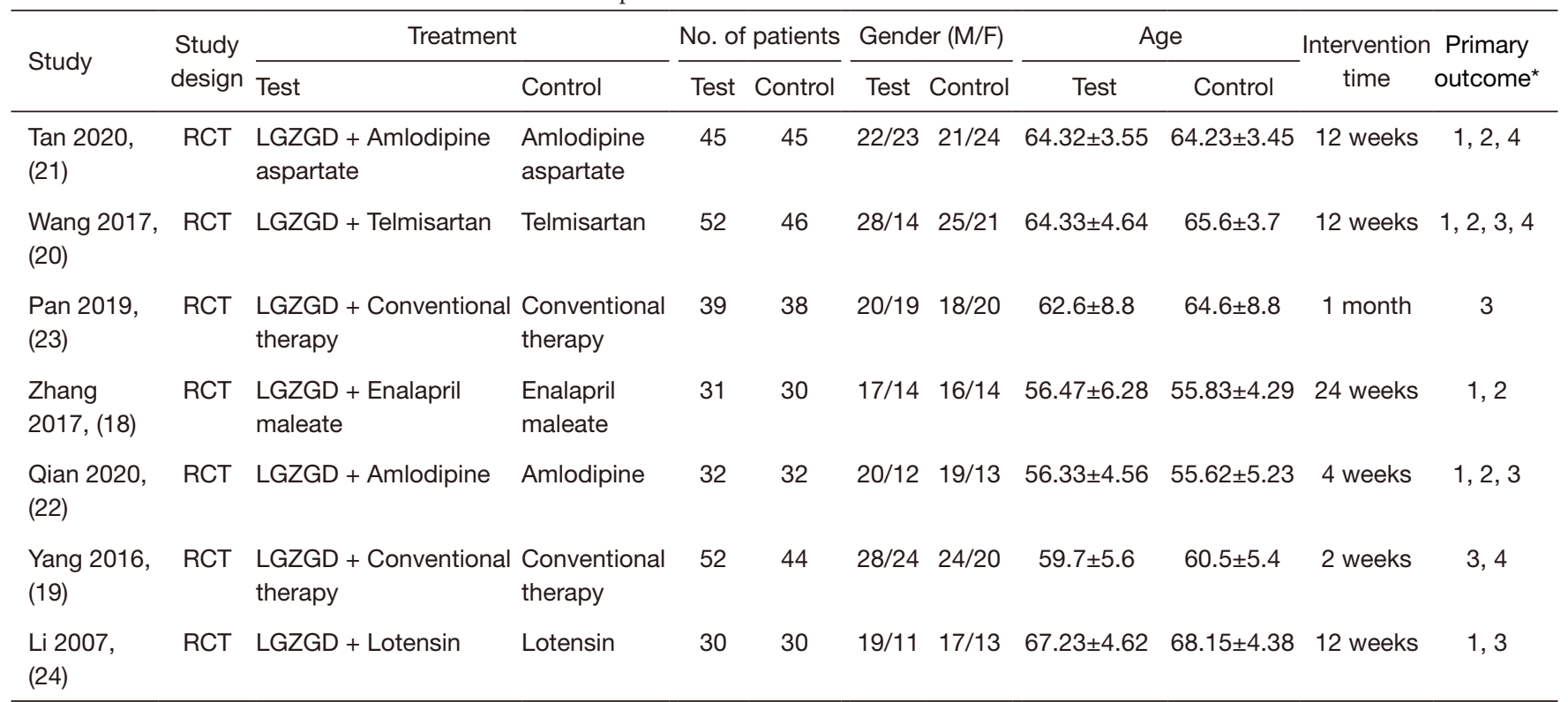

*: 1, effective ratio; 2, SBP change; 3, DBP change; 4, adverse reactions. RCT, randomized controlled trial; SBP, systolic blood pressure; DBP, diastolic blood pressure; LGZDG, Lingguizhugan decoction.

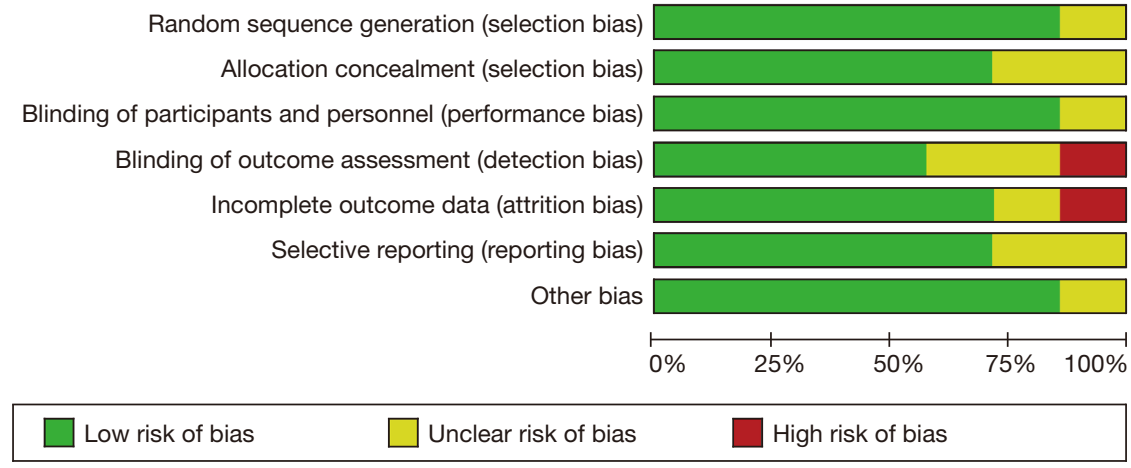

Figure 2 Quality assessment of the studies in this meta-analysis of high, low, and unclear risk of bias are marked in red, green, and yellow.

treatment ranged from 2 to 24 weeks. Outcomes of interests included: SBP change, DBP change, effective ratio, and adverse reactions.

The antihypertensive effect was determined according to the relevant standards in the guiding principles for clinical research of new drugs of TCM (25): (I) strongly effective: after treatment, DBP decreased more than $10 \mathrm{mmHg}$, $\mathrm{BP}$ returned to normal reference range; (II) Moderately effective: after treatment, DBP decreased to a certain extent, but the decrease was less than $10 \mathrm{mmHg}$, and the $\mathrm{BP}$ returned to the normal reference range; (III) ineffective: SBP and DBP did not improve significantly after treatment,
$\mathrm{SBP}>140 \mathrm{mmHg}$. The effective ratio in our outcome variable was the sum of strongly effective and moderate effective ratio.

\section{Results of quality assessment}

Direct comparisons and risk of bias assessment were calculated by Cochrane risk of bias assessment tool. Among the included studies, high risk of detection bias and attrition bias was found in two different studies (Figure 2). A summary of the risk of bias assessment for each study was presented in Figure 3. 


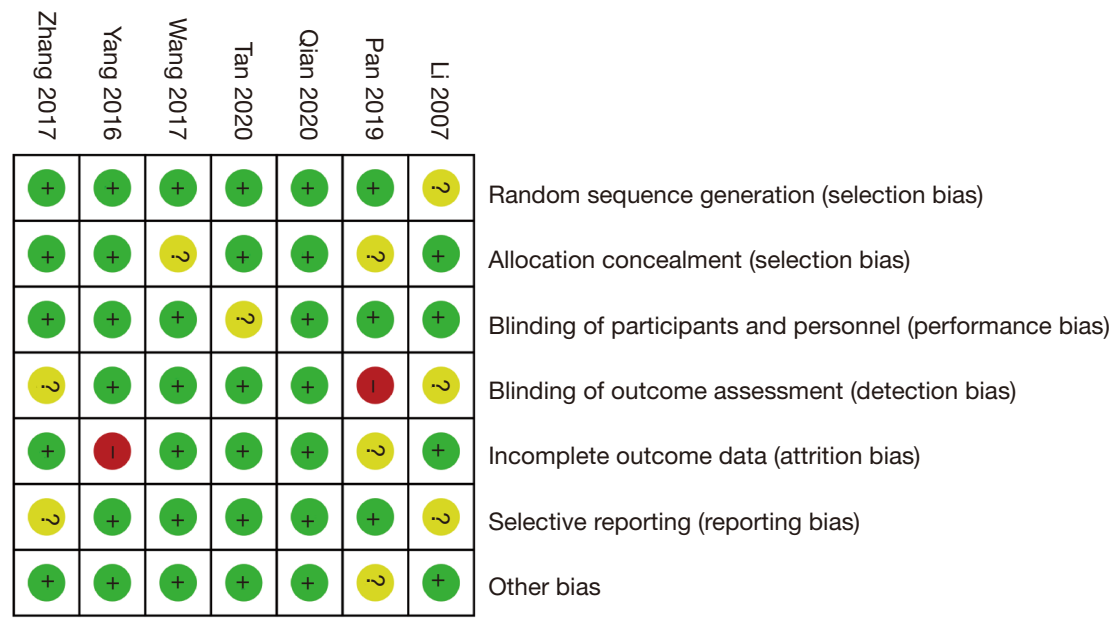

Figure 3 Summary of risk of study bias for individual studies.

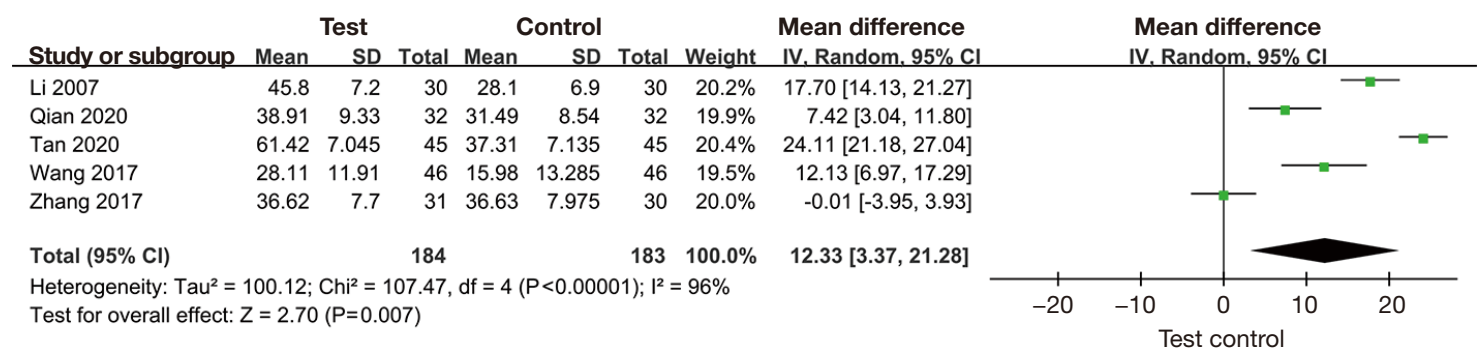

Figure 4 Forest plot evaluating the outcomes of SBP change. SBP, systolic blood pressure; SD, standard deviation; CI, confidence interval.

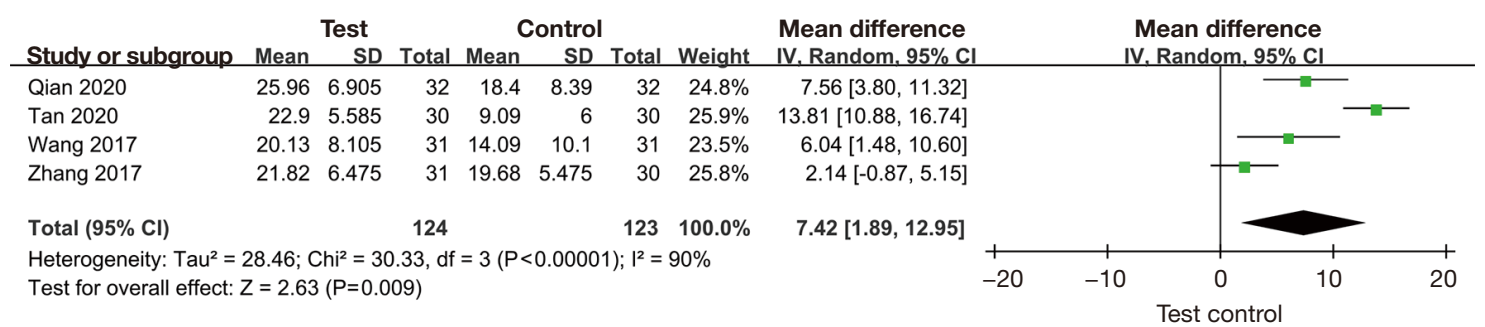

Figure 5 Forest plot evaluating the outcomes of DBP change. DBP, diastolic blood pressure; SD, standard deviation; CI, confidence interval.

\section{Results of heterogeneity test}

A total of 5 studies comprising 184 test group and 183 control group participants reported the SBP change. To analyze the difference of SBP change between two groups, we performed a meta-analysis by using the random effects model based on heterogeneity analysis. The $\mathrm{MD}$ was $12.33 \mathrm{mmHg}$ with $95 \%$ CI: 3.37 to $21.28, \mathrm{P}=0.007$, and significant heterogeneity was found $\left(\mathrm{I}^{2}=96 \%, \mathrm{P}<0.00001\right)$ (Figure 4).
A total of 4 studies reported the DBP change. A random effects model was used to evaluate the heterogeneity of DBP change due to the significant heterogeneity among included studies $\left(\mathrm{I}^{2}=90 \%, \mathrm{P}<0.00001\right)$ (Figure 5), and the result showed that test group had a better reduction of DBP than control group (MD was $7.42 \mathrm{mmHg}$ with $95 \% \mathrm{CI}: 1.89$ to $12.95, \mathrm{P}=0.009)$.

In the evaluation of difference of effective ratio between 


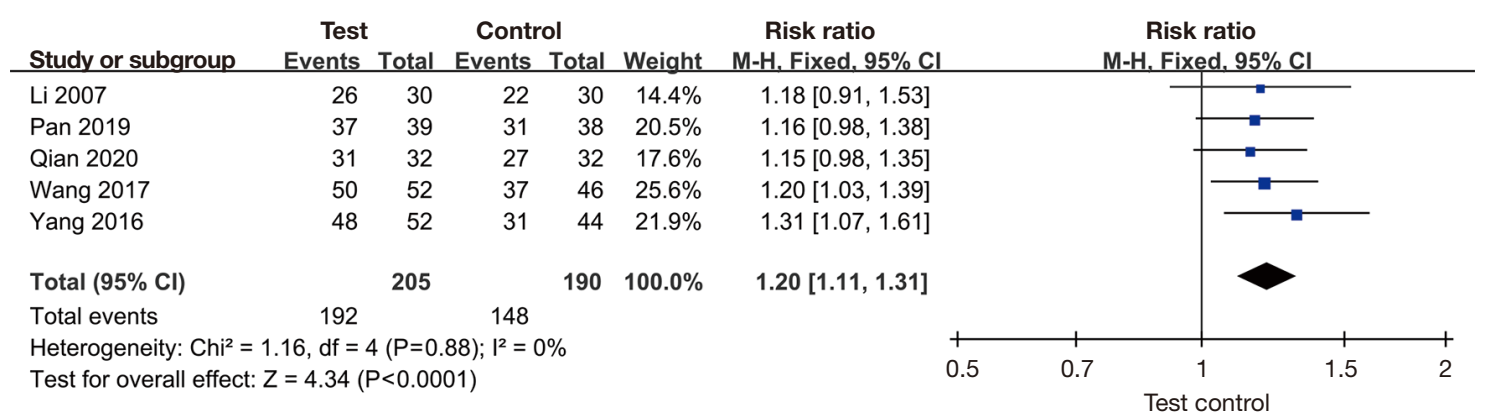

Figure 6 Forest plot evaluating the outcomes of effective ratio. SD, standard deviation; CI, confidence interval.

\begin{tabular}{|c|c|c|c|c|c|c|c|c|c|c|c|}
\hline Study or subgroup & $\begin{array}{l}\text { Test } \\
\text { Events }\end{array}$ & Total & $\begin{array}{l}\text { Contr } \\
\text { Events }\end{array}$ & & Weight & $\begin{array}{l}\text { Risk ratio } \\
\text { M-H, Fixed, } 95 \% \mathrm{Cl}\end{array}$ & & $\begin{array}{r}\text { Risk } \\
\text { M-H, Fixe }\end{array}$ & ratio & & \\
\hline Tan 2020 & 6 & 45 & 7 & 45 & $20.7 \%$ & $0.86[0.31,2.35]$ & & $\longrightarrow$ & & & \\
\hline Wang 2017 & 5 & 52 & 12 & 46 & $37.7 \%$ & $0.37[0.14,0.97]$ & & 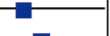 & & & \\
\hline Yang 2016 & 7 & 52 & 13 & 44 & $41.6 \%$ & $0.46[0.20,1.04]$ & & & & & \\
\hline Total $(95 \% \mathrm{Cl})$ & & 149 & & 135 & $100.0 \%$ & $0.51[0.30,0.86]$ & & & & & \\
\hline Total events & 18 & & 32 & & & & & & & & \\
\hline \multicolumn{7}{|c|}{$\begin{array}{l}\text { Heterogeneity: } \mathrm{Chi}^{2}=1.52, \mathrm{df}=2(\mathrm{P}=0.47) ; \mathrm{I}^{2}=0 \% \\
\text { Test for overall effect: } Z=2.54(P=0.01)\end{array}$} & 0.10 .2 & 0.5 & 2 & & 10 \\
\hline
\end{tabular}

Figure 7 Forest plot evaluating the outcomes of adverse reactions. SD, standard deviation; CI, confidence interval.

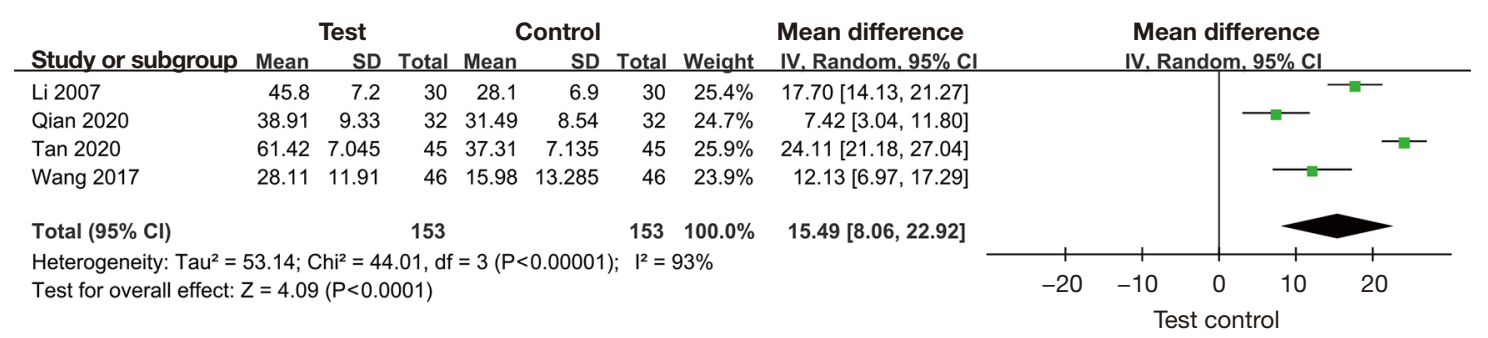

Figure 8 Sensitivity analysis: forest plots of SBP change. SBP, systolic blood pressure; SD, standard deviation; CI, confidence interval.

two groups, 5 articles involving 395 participants were included. The pooled analysis showed that the test group had a better level of effective ratio when compared to control group (RR: 1.20, 95\% CI: 1.11 to 1.31 ), $\mathrm{P}<0.0001$, fixed effect model), without significant heterogeneity $\left(\mathrm{I}^{2}=0 \%, \mathrm{P}=0.88\right.$ ) (Figure 6).

A total of three studies reported the adverse reactions. The forest plot showed that the rate of adverse reactions in test group was lower than in control group (RR: 0.51, $95 \%$ CI: 0.30 to $0.86, \mathrm{P}=0.01$, fixed effects model), without significant heterogeneity among studies $\left(\mathrm{I}^{2}=0 \%, \mathrm{P}=0.47\right)$ (Figure 7).

\section{Results of sensitivity analysis and publication bias}

The included studies were excluded one by one for sensitivity analysis. For SBP, the $\mathrm{I}^{2}$ changed from $96 \%$ to $93 \%$ when Zhang 2017 (18) was excluded, the overall MD did not change significantly after sensitivity analysis ( $M D=15.49, \mathrm{P}<0.0001$ ) (Figure 8), which explained that the heterogeneity among studies was relatively steady.

The funnel plots were primarily drawn to evaluate potential publication bias for the outcomes, which revealed the shapes of the funnel plot with some evidence of symmetry (Figure 9), and the Egger test was non-significant $(\mathrm{P}=0.58)$. 


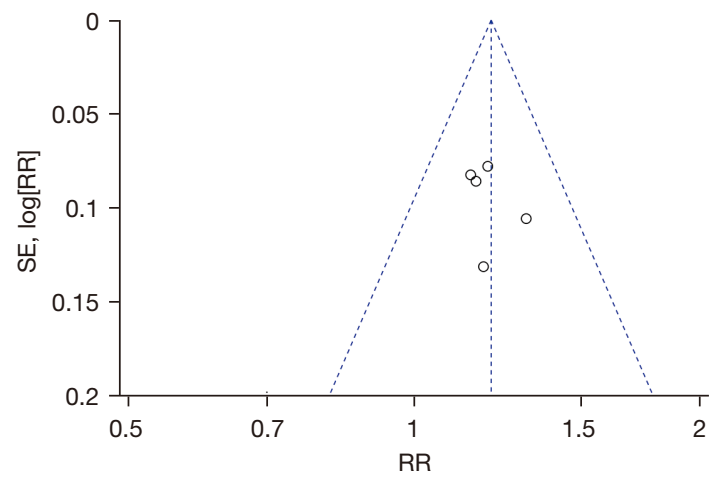

Figure 9 Funnel plot shows no publication bias among studies. $\mathrm{RR}$, risk raito; $\mathrm{SE}$, standard error.

\section{Discussion}

Most patients with hypertension have different degrees of abnormal glucose and lipid metabolism and insulin resistance, which are closely related to cardiovascular and cerebrovascular diseases (3). Western medicine mainly focuses on simple hypertension, with 1 drug corresponding to 1 hypertension mechanism, and there are many potential adverse reactions associated with these drugs $(26,27)$. The use of TCM regulates water and fluid metabolism, reduces weight, and improves obesity, so as to control BP $(28,29)$; however, its specific antihypertensive mechanism needs to be further studied (30).

In TCM, it is believed that hypertension is closely related to patients' congenital endowment deficiency, physical deficiency, advanced age, improper diet, excessive work and leisure, and unregulated emotions (31). In those affected by hypertension, the spleen has lost the power of circulation, the metabolism of water is impaired, fluid accumulates in the body, and forms physical deficiency and obesity. Phlegm dampness arises and stagnates, turbid phlegm accumulates, and over the long-term transforms into heat, phlegm, and blood stasis, which together block the vessels, and result in vertigo (32). Therefore, the principle of TCM in the treatment of hypertension is to strengthen the spleen and help transport and dispel phlegm $(33,34)$.

The ingredients of Lingguizhugan decoction are Poria cocos, Cinnamon Twig, Atractylodes macrocephala, and licorice. Poria cocos is sweet, light, and beneficial to water, which tonifies the spleen. Atractylodes macrocephala assists Poria cocos in tonifying spleen $(35,36)$. Roasted licorice helps Cinnamon twig to support the heart and protect spleen and stomach at the same time. Modern pharmacological research shows that Poria cocos can reduce the contraction amplitude and tension of smooth muscle, affect metabolism in vivo, reduce BP, and reduce blood glucose. Atractylodes macrocephala can promote the generation of endogenous vasodilator substances, inhibit the release of vasoconstrictor substances, and play a role in reducing $\mathrm{BP}(37,38)$. Licorice can expand blood vessels, reduce $\mathrm{BP}$, improve microcirculation, regulate blood glucose, reduce blood lipid level, and inhibit arteriosclerosis (39).

A total of 7 studies were included in our systematic evaluation. The results of meta-analysis showed that the total effective ratio of Lingguizhugan decoction in the adjuvant treatment of hypertension on the basis of routine western medicine treatment was higher than that of western medicine alone (RR: $1.20,95 \%$ CI: 1.11 to 1.31 , $\mathrm{P}<0.0001$ ), suggesting that Lingguizhugan decoction has a good adjuvant effect on hypertension. In addition, it was more obvious than the control group in reducing SBP (MD: $12.33 \mathrm{mmHg}, 95 \% \mathrm{CI}: 3.37$ to $21.28, \mathrm{P}=0.007)$ and $\mathrm{DBP}$ (MD: $7.42 \mathrm{mmHg}, 95 \% \mathrm{CI}: 1.89$ to $12.95, \mathrm{P}=0.009$ ), and had fewer adverse reactions (RR: $0.51,95 \%$ CI: 0.30 to 0.86 , $\mathrm{P}=0.01)$. In terms of improving the curative effect of clinical symptoms, the Lingguizhugan decoction combined with western medicine group performed better than the simple western medicine group.

Some literature also mentioned the influence of Lingguizhugan decoction on other health indicators. For example, Yang's study (19) mentioned that Lingguizhugan decoction can increase the heart output, increase left ventricular mass index, reduce pulmonary artery pressure, and relieve the patient's pulmonary hypertension. Tan's research (21) mentioned that Lingguizhugan decoction can regulate lipid metabolism and insulin resistance, protect myocardial cells, and regulate body fluid metabolism.

Western medicine was aimed at lowering blood pressure, and one drug corresponded to one hypotension mechanism, and there were many side effects at the same time. Chinese medicine regulated body fluid metabolism, reduces weight and improved obesity (37). Lingguizhugan decoction combined with western medicine was used to treat hypertension, which was beneficial to improve the clinical symptoms of patients, protected the kidneys, regulated blood pressure and blood lipid levels, and enhance the therapeutic effect (34).

This meta-analysis had some limitations. Firstly, because Lingguizhugan decoction is a unique traditional 
prescription in China, the reports published were mainly in Chinese, and the quality was relatively poor. Secondly, the number of included studies was small, and the sample size was relatively low, lacking the demonstration of large sample size. Thirdly, due to the limitation of the number of included literatures, as the combined western medicine scheme used in the included literatures either conventional therapy or amlodipine, we cannot compare the differences between different combination therapy.

In conclusion, Lingguizhugan decoction combined with western medicine can reduce patients' blood pressure and improve their clinical symptoms, with high safety. This study was limited by the quantity and quality of the included studies, thus the findings require verification through further large sample and multi center follow-up controlled trials.

\section{Acknowledgments}

Funding: This work was supported by Special project on prevention and treatment of diseases in Guangdong Provincial Hospital of Traditional Chinese Medicine (No. YN2019ZWB05).

\section{Footnote}

Reporting Checklist: The authors have completed the PRISMA reporting checklist. Available at https://dx.doi. org/10.21037/apm-21-2639

Conflicts of Interest: All authors have completed the ICMJE uniform disclosure form (available at https://dx.doi. org/10.21037/apm-21-2639). The authors have no conflicts of interest to declare.

Ethical Statement: The authors are accountable for all aspects of the work in ensuring that questions related to the accuracy or integrity of any part of the work are appropriately investigated and resolved.

Open Access Statement: This is an Open Access article distributed in accordance with the Creative Commons Attribution-NonCommercial-NoDerivs 4.0 International License (CC BY-NC-ND 4.0), which permits the noncommercial replication and distribution of the article with the strict proviso that no changes or edits are made and the original work is properly cited (including links to both the formal publication through the relevant DOI and the license).
See: https://creativecommons.org/licenses/by-nc-nd/4.0/.

\section{References}

1. Mills KT, Bundy JD, Kelly TN, et al. Global Burden of Hypertension. J Hypertens 2015;33:e2.

2. Bao M, Wang $\mathrm{L}$. The longitudinal trend of hypertension prevalence in Chinese adults from 1959 to 2018: a systematic review and meta-analysis. Ann Palliat Med 2020;9:2485-97.

3. Galiè N, Humbert M, Vachiery JL, et al. 2015 ESC/ERS Guidelines for the diagnosis and treatment of pulmonary hypertension: The Joint Task Force for the Diagnosis and Treatment of Pulmonary Hypertension of the European Society of Cardiology (ESC) and the European Respiratory Society (ERS): Endorsed by: Association for European Paediatric and Congenital Cardiology (AEPC), International Society for Heart and Lung Transplantation (ISHLT). Eur Heart J 2016;37:67-119.

4. Mansia G, De Backer G, Dominiczak A, et al. 2007 ESH-ESC Guidelines for the management of arterial hypertension: the task force for the management of arterial hypertension of the European Society of Hypertension (ESH) and of the European Society of Cardiology (ESC). Blood Press 2007;16:135-232.

5. de Franchis R; Baveno VI Faculty. Expanding consensus in portal hypertension: Report of the Baveno VI Consensus Workshop: Stratifying risk and individualizing care for portal hypertension. J Hepatol 2015;63:743-52.

6. Chobanian AV. Guidelines for the Management of Hypertension. Med Clin North Am 2017;101:219-27.

7. Thomas PR, Nash GB, Dormandy JA. White cell accumulation in dependent legs of patients with venous hypertension: a possible mechanism for trophic changes in the skin. Br Med J (Clin Res Ed) 1988;296:1693-5.

8. Symplicity HTN-2 Investigators; Esler MD, Krum $\mathrm{H}$, et al. Renal sympathetic denervation in patients with treatment-resistant hypertension (The Symplicity HTN-2 Trial): a randomised controlled trial. Lancet 2010;376:1903-9.

9. Beswick RA, Zhang H, Marable D, et al. Long-term antioxidant administration attenuates mineralocorticoid hypertension and renal inflammatory response. Hypertension 2001;37:781-6.

10. Wu CX, Liu Y, Zhang JC. Chronic intermittent hypoxia and hypertension: a review of systemic inflammation and Chinese medicine. Chin J Integr Med 2013;19:394-400.

11. Wu A, Zhang D, Zhai J. Relationship between Traditional 
Chinese Medicine Syndrome, hsCRP, oxLDL and Cerebral Infarction in Patients with Hypertension. Chinese Journal of Integrative Medicine on Cardio-/ Cerebrovascular Disease 2013;15:34-6.

12. Liu MT, Huang YJ, Zhang TY, et al. Lingguizhugan decoction attenuates diet-induced obesity and hepatosteatosis via gut microbiota. World J Gastroenterol 2019;25:3590-606.

13. Fang HY, Huang JL, FF S, et al. Effects of Linggui Zhugan Decoction on Levels of Angiotensin II, Endothelin-1, Tumor Necrosis Factor- $\alpha$ and Interleukin$1 \beta$ in Rats with Chronic Heart Failure. Journal of Anhui Traditional Chinese Medical College 2010;12:322-4.

14. Chen DS, Ke B, Huang YJ, et al. Effects of the modified linggui zhugan decoction (see text) combined with short-term very low calorie diets on glycemic control in newly diagnosed type 2 diabetics. J Tradit Chin Med 2011;31:185-8.

15. Wang $Y$, Jin $M$, Zhang L, et al. Effect of a combination of calorie-restriction therapy and Lingguizhugan decoction on levels of fasting blood lipid and inflammatory cytokines in a high-fat diet induced hyperlipidemia rat model. J Tradit Chin Med 2015;15:45-8.

16. Wang L, Hou XY, Huang JL, et al. Effect of Linggui Zhugan Decoction on TNF- $\alpha$ in myocardial tissue and $\mathrm{NF}-\kappa \mathrm{B}$ and IL- $1 \beta$ in serum of model rats with chronic heart failure. Chinese Traditional \& Herbal Drugs 2013;44:590-4.

17. Yao L, Wei J, Shi S, et al. Modified lingguizhugan decoction incorporated with dietary restriction and exercise ameliorates hyperglycemia, hyperlipidemia and hypertension in a rat model of the metabolic syndrome. BMC Complement Altern Med 2017;17:132.

18. Zhang Y, Rui J, Jia B, et al. Modified Lingguizhugan decoction combined with western medicine in the treatment of 31 cases of left ventricular hypertrophy in essential hypertension. TCM Res 2017;30:20-2.

19. Yang L, Zhang Z, Jiang H, et al. Clinical Effects of Lingguizhugan Decoction in the Treatment of Hypoxic Pulmonary Hypertension. Chinese General Medicine 2016;19:3495-9.

20. Wang J, Li S, Xie X, et al. Therapeutic effect of modified Lingguizhugan decoction combined with telmisartan on senile obese hypertension. Modern Journal of Integrated Traditional Chinese and Western Medicine 2017;26:1898-900.

21. Tan Q. Clinical study on the treatment of hypertension with Lingguizhugan decoction modified aspartic acid tablets. Journal of Integrated Traditional Chinese and Western Medicine 2020;20:137-8.

22. Qian H, Zhang H. Clinical effect of modified Linggui Zhugan decoction in the treatment of obesity hypertension. Clinical Medical Research and Practice 2020;5:137-8.

23. Pan Q, Tian Z, Chen C. Effect of Linggui Zhugan Decoction on carotid intima-media thickness in patients with phlegm dampness hypertension complicated with lacunar cerebral infarction. Yunnan Journal of Traditional Chinese Medicine 2019;40:40-1.

24. Li S, Wei D. Therapeutic effect of Lingguizhugan Decoction on simple systolic hypertension in the elderly. Hubei Journal of Traditional Chinese Medicine 2007;29:27-28.

25. Wang LY, Li Y, Ding Y, et al. Research progress of TCM syndrome dynamics of hypertension. Hebei Journal of Traditional Chinese Medicine 2010;32:3.

26. Berzigotti A . Advances and challenges in cirrhosis and portal hypertension. BMC Medicine 2017;15:200.

27. Huang S, Chu J, Lu ZL, et al. Evaluation on the effectiveness of community intervention on hypertension related knowledge in mid-western rural area of Shandong province. Zhonghua Yu Fang Yi Xue Za Zhi 2011;45:1007-11.

28. Wang J, Xiong X, Liu W. Traditional chinese medicine syndromes for essential hypertension: a literature analysis of 13,272 patients. Evid Based Complement Alternat Med 2014;2014:418206.

29. Tou WI, Chen CY. Traditional Chinese medicine as dual guardians against hypertension and cancer? J Biomol Struct Dyn 2012;30:299-317.

30. Xiong X, Yang X, Liu W, et al. Trends in the treatment of hypertension from the perspective of traditional chinese medicine. Evid Based Complement Alternat Med 2013;2013:275279.

31. Jie W, Xiong X. Control Strategy on Hypertension in Chinese Medicine. Evidence-Based Complementray and Alternative Medicine 2011;12:284-8.

32. Jie W, Xiong X. Evidence-Based Chinese Medicine for Hypertension. Evid-Based Compl Alt 2013;9:97-8.

33. Zou P. Traditional Chinese Medicine, Food Therapy, and Hypertension Control: A Narrative Review of Chinese Literature. Am J Chin Med 2016;44:1579-94.

34. Zhu YB, Wang Q, Deng QW, et al. Relationships between constitutional types of traditional Chinese medicine and hypertension. Zhong Xi Yi Jie He Xue Bao 2010;8:40-5. 35. Wang YY, Jin MH, Ke B, et al. Effects of linggui zhugan 
decoction combined calorie restriction on the insulin resistance of model rats and mechanisms research. Zhongguo Zhong Xi Yi Jie He Za Zhi 2013;33:356-60.

36. Song ZH, Dai SJ, Li HQ, et al. Study on the compatibility of composite herbal medicines of the lingguizhugan decoction. Zhongguo Zhong Yao Za Zhi 2002;27:760-2.

37. Huang J, Wang L, Shi H, et al. Effect of Lingguizhugan decoction on myocardial Nuclear factor kappa B protein expression in rats with chronic heart failure. J Tradit Chin Med 2013;33:343-8.

38. Li TH, Hou CC, Chang LT, et al. Anti-Hyperglycemic

Cite this article as: Xie P, Li X, Jiang H, Qiu S, Liang Y. Clinical effects of Lingguizhugan decoction in the treatment of hypertension: a systematic review and meta-analysis. Ann Palliat Med 2021;10(11):11529-11538. doi: 10.21037/apm-21-2639
Properties of Crude Extract and Triterpenes from Poria cocos. Evid Based Complement Alternat Med 2011;2011:128402.

39. Liu T, Yang LL, Zou L, et al. Chinese medicine formula lingguizhugan decoction improves Beta-oxidation and metabolism of Fatty Acid in high-fat-diet-induced rat model of Fatty liver disease. Evid Based Complement Alternat Med 2013;2013:429738.

(English Language Editor: J. Jones) 